\title{
Prediction of Forming Limit Diagrams under combined Bending- Stretching loadings
}

\author{
Mohamed Ben Bettaieb ${ }^{1,2, a}$, Honoré Lagaza ${ }^{1}$, Farid Abed-Meraim ${ }^{1,2}$, and Xavier Lemoine ${ }^{1}$ \\ ${ }^{1}$ LEM3, UMR CNRS 7239 - Arts et Métiers ParisTech, 4 rue Augustin Fresnel, 57078 Metz Cedex 3, France \\ ${ }^{2}$ DAMAS, Laboratory of Excellence on Design of Alloy Metals for low-mAss Structures, Université de Lorraine, France
}

\begin{abstract}
A number of parts and components involved in the automotive industry are made of thin bent sheets. Unfortunately, the classical predictions based on traditional Forming Limit Diagrams are not relevant when the strain distribution is heterogonous over the thickness, as is the case for bent sheet metals. The aim of the present contribution is to propose an extension of the well-known Marciniak-Kuczynski approach to account for the effect of bending on formability prediction. The new developed tool allows predicting the limit strains for the whole range of strain paths. The mechanical behavior of the studied sheets follows the rigid-plastic flow theory. Through numerical results, it is shown that bending tends to decrease the formability limit of the sheet metal.
\end{abstract}

\section{Introduction}

The study of ductility of metallic components and parts is an ambitious challenge in academic and industrial applications. This ductility is often characterized by a Forming Limit Diagram (FLD). The FLD concept has been initially introduced by Keeler and Backofen [1], for representing the limit strains in the range of positive strain paths, and has been extended by Goodwin [2] to the whole range of strain paths. Due to the complexity of the experimental characterization of FLDs and its relatively high cost, a number of theoretical and / or numerical models have been developed in the literature. These models generally require the use of an instability criterion along with a constitutive law to describe the evolution of the mechanical state of the studied sheet. In the literature, a large amount of models have been developed to numerically determine FLDs.

From these theoretical /numerical approaches, we can quote the work of Marciniak and Kuczynski [4], who coupled the initial imperfection approach with a rigidplastic flow theory in order to predict the limit strains in the range of positive strain paths. Later, Hutchinson and Neale [5] extended the initial imperfection approach, initially developed in [4], to the range of negative strain paths. In the latter work, both the rigid-plastic flow theory and the deformation theory of plasticity were used to model the mechanical behavior of the studied sheet. In most of the approaches proposed in the past, the effect of bending deformation on formability is not taken into account, since homogeneous deformation through the sheet thickness is assumed. However, it is not uncommon that metallic sheets used in industrial applications

\footnotetext{
a Corresponding author: Mohamed.BenBettaieb@ensam.eu
}

(automotive, aeronautic...) undergo combination of bending and stretching deformations. Such modes of deformation may have a substantial impact on the formability of the studied parts. Several experimental studies revealed that bending tends to increase the formability of metal sheets. Nowadays, there are many attempts to develop FLD prediction models that take into account bending effects. In this field, Shi and Gerdeen [6] integrated the effect of strain gradient and curvature in the prediction of FLDs for anisotropic materials. Sriram et al. [7] developed an empirical model to characterize fracture behavior during forming of advanced high strength steels under bending dominated conditions. He et al. [8] extended the initial imperfection approach developed in [4] to the case of combined stretching-bending loading.

Nonetheless, their work was restricted to the righthand side of the FLD, where bending is along the major stretch direction. Two different constitutive laws were used in [8] to model the mechanical behavior of the sheet: the flow theory and the deformation theory of plasticity. The obtained numerical results show that the bending process decreases sheet metal formability when the flow theory is used, whereas the opposite trend is observed with the deformation theory. Furthermore, the model developed in [8] was combined with finite element analysis in order to predict the forming limits of a sheet metal undergoing continuous bending-under-tension loading [8]. More recently, Safdarian [10] developed a new model for predicting FLDs of tailor welded blanks. In this model, the $\mathrm{M}-\mathrm{K}$ approach developed in [5] has been enriched by taking into account the effect of 
bending strain on sheet metal formability. The flow theory of plasticity was used to describe the mechanical behavior of the studied sheet. Contrary to the approach developed in [8], the modified $\mathrm{M}-\mathrm{K}$ model developed in [10] allows predicting the limit strains for the whole range of strain paths. However, this model presents some drawbacks and limitations. Indeed, in this model, the strain paths, both within the band and outside it, are assumed to remain linear during deformation. Consequently, the increment of strain components is replaced by total strain components in the constructive equations governing the associated modified $\mathrm{M}-\mathrm{K}$ model. This assumption is not relevant in the band zone, where it is known that the strain path is constantly changing along deformation. Besides, the shear stresses have been withdrawn in the formulation of the equilibrium equations. This point represents the second limitation of the model. Indeed, the shear stresses cannot be neglected, especially when the band is not perpendicular to the major strain direction. In the present paper, a new modified $\mathrm{M}-\mathrm{K}$ model is developed to address the abovementioned issues. The main result of this paper is that the addition of bending effects tends to decrease sheet metal formability for the whole range of strain paths. This result confirms the numerical predictions reported in [8].

The remainder of the paper is organized as follows:

- The equations governing the new modified $\mathrm{M}-\mathrm{K}$ model will be detailed in the second section.

- In the third section, the numerical and algorithmic aspects related to the model will be presented.

- Various numerical results obtained by application of the developed model will be presented and discussed in the fourth section.

\section{Notations}

The following notations and abbreviations are adopted in this paper:

- $\bullet^{\mathrm{T}}$ : transpose of tensor $\bullet$.

$\bullet^{\mathrm{B}}$ : quantity $\bullet$ associated with the band.

$-\bullet^{\mathrm{H}}$ : quantity $\bullet$ associated with the zone located outside the band.

- $\bullet_{\mathrm{ST}}$ : the stretching part of quantity $\bullet$.

- $\bullet_{\mathrm{BE}}$ : the bending part of quantity $\bullet$.

$-\bullet(t)$ : value of quantity $\bullet$ at time $t$.

$-\bullet_{i}$ : quantity $\bullet$ associated with the integration point $i$.

These notations can be combined. For instance, the stretching part of the strain tensor in the band is denoted by $\boldsymbol{\varepsilon}_{\mathrm{ST}}^{\mathrm{B}}$.

\section{Constitutive equations}

\subsection{Mechanical behavior}

Elasticity is neglected in the subsequent constitutive equations. This assumption is justified, because strain localization occurs at relatively large strains. Moreover, the plastic flow is assumed to be isotropic and incompressible. The rigid-plastic flow theory is then used to model the mechanical behavior of the studied sheet metal. Hence, the strain rate tensor $\dot{\boldsymbol{\varepsilon}}$ is derived by using the normality flow rule

$$
\dot{\boldsymbol{\varepsilon}}=\dot{\lambda} \frac{\partial \sigma_{\mathrm{eq}}}{\partial \boldsymbol{\sigma}},
$$

where $\dot{\lambda}$ is the plastic multiplier (equal here to the von Mises equivalent strain rate $\dot{\varepsilon}_{\text {eq }}$ ), $\boldsymbol{\sigma}$ is the Cauchy stress tensor, and $\sigma_{\text {eq }}$ is the von Mises equivalent stress. By using the definition of $\sigma_{\text {eq }}$, the rigid-plastic constitutive law can be derived from Eq. (1)

$$
\dot{\boldsymbol{\varepsilon}}=\frac{3 \dot{\varepsilon}_{\mathrm{eq}}}{2 \sigma_{\mathrm{eq}}} \mathbf{S} \Leftrightarrow \mathbf{S}=\frac{2 \sigma_{\mathrm{eq}}}{3 \dot{\varepsilon}_{\mathrm{eq}}} \dot{\boldsymbol{\varepsilon}},
$$

where $\mathbf{S}$ is the deviatoric part of $\boldsymbol{\sigma}$. The two latter tensors are linked by the following relation:

$$
\boldsymbol{\sigma}=\mathbf{S}+p \mathbf{I}_{2}
$$

where $p$ is the hydrostatic pressure equal to $1 / 3 \operatorname{tr}(\boldsymbol{\sigma})$ and $\mathbf{I}_{2}$ is the second-order identity tensor.

The equivalent stress $\sigma_{\mathrm{eq}}$ is related to the equivalent strain $\varepsilon_{\text {eq }}$ by the Hollomon isotropic hardening law

$$
\sigma_{\text {eq }}=K \varepsilon_{\text {eq }}^{n}
$$

where $K$ et $n$ are two material hardening parameters.

By combining Eqs. (2) and (4) one obtains

$$
\mathbf{S}=\frac{2 K \varepsilon_{\mathrm{eq}}^{n}}{3 \dot{\varepsilon}_{\mathrm{eq}}} \dot{\boldsymbol{\varepsilon}} .
$$

\subsection{Modified M-K approach}

\subsubsection{Assumptions related to the Bending-Stretching process}

The sheet is assumed to be initially flat, with $X$ and $Y$ the two in-plane principal directions and $Z$ the throughthickness direction, as illustrated in Figure 1. It should be understood that the $X-Y-Z$ coordinate system represents a material coordinate frame, which will rotate as material deforms. The following assumptions and choices are made in the development of this modified $\mathrm{M}-\mathrm{K}$ approach:

- The sheet is first subjected to a bending moment $M$ applied in $Y$-direction, resulting in the sheet being curved along $X$-direction, with a uniform curvature $R$. Hence, there is a single curvature along $X$-direction, while the sheet remains flat along the $Y$-direction (see, Figure 1).

- The neutral axis is assumed to remain at the middle layer of the sheet metal during deformation. 
- The studied sheet is assumed to be wide enough (along the $Y$-direction) relative to its thickness. Consequently, the strain component $\varepsilon_{\mathrm{yy}}$ can be neglected during the bending process.

- After the bending stage, an axial major strain along $X$ and a minor strain along $Y$ are then applied to the sheet under a constant strain path $\rho$, while the bending radius is kept constant equal to $R$.

- The studied sheet is assumed to be thin. Therefore, the plane-stress assumption is adopted, in accordance with several literature works [7]. This assumption can be expressed in the following index form:

$$
\sigma_{\mathrm{xz}}=\sigma_{\mathrm{yz}}=\sigma_{\mathrm{zz}}=0
$$

This plane-stress condition allows us to determine the hydrostatic pressure $p$ introduced in Eq. (3)

$$
p=S_{\mathrm{xx}}+S_{\mathrm{yy}}
$$

Combining the plane-stress and the incompressibility conditions, one can easily obtain

$$
\varepsilon_{\mathrm{xz}}=\varepsilon_{\mathrm{yz}}=0 \quad ; \quad \varepsilon_{\mathrm{zz}}=-\left(\varepsilon_{\mathrm{xx}}+\varepsilon_{\mathrm{yy}}\right)
$$

Equations (6)-(8) are valid for any material point of the sheet.

\subsubsection{Modified $M-K$ equations}

The $\mathrm{M}-\mathrm{K}$ approach is based on the assumption of the preexistence of an initial geometric imperfection in the form of a band across the thickness of the sheet, as illustrated in Figure 1. This band is defined by its normal unit vector $\overrightarrow{\boldsymbol{n}}$. The angle between vector $\overrightarrow{\boldsymbol{n}}$ and the major strain direction $X$ is denoted $\theta$. The initial thickness of the band (resp. zone outside the band) is equal to $h_{0}^{\mathrm{B}}$ (resp. $\left.h_{0}^{\mathrm{H}}\right)$.

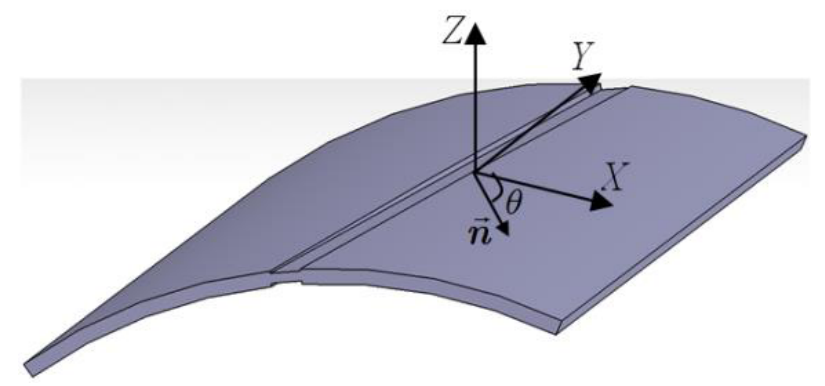

Figure 1. Initial imperfection approach for the sheet metal

As a consequence of the assumptions made in Section 2.2.1, it can be shown that the different mechanical variables (strain, stress...) are independent of the $x$ and $y$ coordinates, both inside and outside the band. They depend only on the $z$ coordinate. Under the incompressibility and plane-stress conditions, it is more convenient to rewrite in what follows the governing equations in the form of an in-plane formulation (in the plane $(X Y))$. The out-of-plane components can be easily derived from Eqs. (6)-(8).
The total strain $\boldsymbol{\varepsilon}$ is additively decomposed into a bending part $\boldsymbol{\varepsilon}_{\mathrm{BE}}$ and a stretching part $\boldsymbol{\varepsilon}_{\mathrm{ST}}$

$$
\boldsymbol{\varepsilon}=\boldsymbol{\varepsilon}_{\mathrm{BE}}+\boldsymbol{\varepsilon}_{\mathrm{ST}}
$$

The above decomposition is valid both inside and outside the band.

However, the bending part $\boldsymbol{\varepsilon}_{\mathrm{BE}}$ of the deformation tensor has the same expression in both zones

$$
\boldsymbol{\varepsilon}_{\mathrm{BE}}=\left(\begin{array}{cc}
\operatorname{Ln}(1+z / R) & 0 \\
0 & 0
\end{array}\right),
$$

where $z$ is the third coordinate of the material point considered.

The stretching part $\dot{\boldsymbol{\varepsilon}}_{\mathrm{ST}}^{\mathrm{H}}$ is assumed to be uniform over the thickness of the sheet and is given by the following expression:

$$
\dot{\boldsymbol{\varepsilon}}_{\mathrm{ST}}^{\mathrm{H}}=\left(\begin{array}{cc}
\dot{\varepsilon}_{\mathrm{ST} \mathrm{xx}}^{\mathrm{H}} & 0 \\
0 & \rho \dot{\varepsilon}_{\mathrm{STxx}}^{\mathrm{H}}
\end{array}\right),
$$

where the strain-path ratio $\rho$ is varied in the range $-1 / 2 \leq \rho \leq 1$ to span the complete FLD.

The stretching part of the strain rate in the band $\dot{\boldsymbol{\varepsilon}}_{\mathrm{ST}}^{\mathrm{B}}$ is related to that outside the band $\dot{\boldsymbol{\varepsilon}}_{\mathrm{ST}}^{\mathrm{H}}$ by the following kinematic compatibility condition:

$$
\dot{\boldsymbol{\varepsilon}}_{\mathrm{ST}}^{\mathrm{B}}=\dot{\boldsymbol{\varepsilon}}_{\mathrm{ST}}^{\mathrm{H}}+(1 / 2)\left((\dot{\overrightarrow{\mathbf{c}}} \otimes \overrightarrow{\boldsymbol{n}})+(\dot{\overrightarrow{\mathbf{c}}} \otimes \overrightarrow{\boldsymbol{n}})^{\mathrm{T}}\right),
$$

where $\dot{\overrightarrow{\mathbf{c}}}$ is the jump vector. Vectors $\dot{\overrightarrow{\mathbf{c}}}$ and $\vec{n}$ are assumed to be uniform over the thickness of the sheet. The evolution of the band orientation $\theta$ is given by the following relation:

$$
\theta=\arctan \left[\tan \left(\theta_{0}\right) \exp (1-\rho) \varepsilon_{\mathrm{ST} \mathrm{xx}}^{\mathrm{H}}\right],
$$

where $\theta_{0}$ is the initial value of the band orientation.

The global equilibrium equation across the band can be expressed as follows:

$$
\left(\int_{-h^{\mathrm{H}} / 2}^{h^{\mathrm{H}} / 2} \boldsymbol{\sigma}^{\mathrm{H}} \mathrm{d} z\right) \cdot \overrightarrow{\boldsymbol{n}}=\left(\int_{-h^{\mathrm{B}} / 2}^{h^{\mathrm{B}} / 2} \boldsymbol{\sigma}^{\mathrm{B}} \mathrm{d} z\right) \cdot \overrightarrow{\boldsymbol{n}},
$$

where $h^{\mathrm{H}}$ and $h^{\mathrm{B}}$ are the current thicknesses of the sheet outside and in the band zone, respectively. They are expressed as functions of the initial thicknesses $h_{0}^{\mathrm{H}}$ and $h_{0}^{\mathrm{B}}$ and of the components of $\dot{\boldsymbol{\varepsilon}}_{\mathrm{ST}}^{\mathrm{H}}$ and $\dot{\boldsymbol{\varepsilon}}_{\mathrm{ST}}^{\mathrm{B}}$

$$
h^{\mathrm{H}}=h_{0}^{\mathrm{H}} \mathrm{e}^{-\left(\varepsilon_{\mathrm{ST} x \mathrm{x}}^{\mathrm{H}}+\varepsilon_{\mathrm{STyy}}^{\mathrm{H}}\right)} \quad ; \quad h^{\mathrm{B}}=h_{0}^{\mathrm{B}} \mathrm{e}^{-\left(\varepsilon_{\mathrm{ST} x \mathrm{x}}^{\mathrm{B}}+\varepsilon_{\mathrm{ST} y y}^{\mathrm{B}}\right)} .
$$

It is difficult to determine an exact value for the two integrals introduced in Eq. (14). Therefore, they are rather numerically approximated by the trapezoidal method, 
after a geometric discretization of the sheet thickness is performed

$$
\begin{aligned}
& \int_{-h^{\mathrm{H}} / 2}^{h^{\mathrm{H}} / 2} \boldsymbol{\sigma}^{\mathrm{H}} \mathrm{d} z=\sum_{i=1}^{i=N_{\mathrm{p}}-1}\left(\frac{\left(z_{i+1}^{\mathrm{H}}-z_{i}^{\mathrm{H}}\right)\left(\boldsymbol{\sigma}_{i+1}^{\mathrm{H}}-\boldsymbol{\sigma}_{\mathrm{i}}^{\mathrm{H}}\right)}{2}\right) \\
& \int_{-h^{B} / 2}^{h^{B} / 2} \boldsymbol{\sigma}^{\mathrm{B}} \mathrm{d} z=\sum_{i=1}^{i=N_{\mathrm{p}}-1}\left(\frac{\left(z_{i+1}^{\mathrm{B}}-z_{i}^{\mathrm{B}}\right)\left(\boldsymbol{\sigma}_{i+1}^{\mathrm{B}}-\boldsymbol{\sigma}_{\mathrm{i}}^{\mathrm{B}}\right)}{2}\right),
\end{aligned}
$$

where $N_{\mathrm{p}}$ is the number of integration points used in the numerical quadrature, which is assumed, for convenience, to be the same for both zones. $z_{i}^{\mathrm{H}}$ (resp. $z_{i}^{\mathrm{B}}$ ) is the current position of the $i^{\text {th }}$ integration point over the thickness in the zone outside the band (resp. in the band). Hence, $z_{1}^{\mathrm{H}}, z_{N_{\mathrm{p}}}^{\mathrm{H}}, z_{1}^{\mathrm{B}}$, and $z_{N_{\mathrm{p}}}^{\mathrm{B}}$ are equal to $-h^{\mathrm{H}} / 2, h^{\mathrm{H}} / 2,-h^{\mathrm{B}} / 2$, and $h^{\mathrm{B}} / 2$, respectively.

Furthermore, $z_{i}^{\mathrm{H}}$ (resp. $z_{i}^{\mathrm{B}}$ ) is related to its initial value $z_{i 0}^{\mathrm{H}}\left(\right.$ resp. $\left.z_{i 0}^{\mathrm{B}}\right)$ by

$$
z_{i}^{\mathrm{H}}=z_{i 0}^{\mathrm{H}} \mathrm{e}^{-\left(\varepsilon_{\mathrm{ST} x \mathrm{H}}^{\mathrm{H}}+\varepsilon_{S T Y y}^{\mathrm{H}}\right)} \quad ; \quad z_{i}^{\mathrm{B}}=z_{i 0}^{\mathrm{B}} \mathrm{e}^{-\left(\varepsilon_{\mathrm{STXx}}^{\mathrm{B}}+\varepsilon_{S T y y}^{\mathrm{B}}\right)} .
$$

\section{Algorithmic aspects}

\subsection{Bending process}

Before the stretching loading is applied, the sheet metal is first bent by applying a bending moment $M$. During this bending stage, the curvature radius decreases from $+\infty$ (which corresponds to a flat sheet) to a finite value $R$. This bending is moderate compared to stretching (typically the ratio $h_{0}^{\mathrm{H}} / R$ does not exceed 0.2 ). Therefore, it is legitimate to consider the distance $z$ of any integration point to the neutral axis as a constant value, which is taken equal to its initial value $z_{0}$. At the end of this bending process, only the equivalent strains corresponding to the different points located inside and outside the band are computed and stored

$$
\forall i=1, \ldots, N_{\mathrm{p}}:\left\{\begin{array}{l}
\varepsilon_{\mathrm{eq} i}^{\mathrm{H}}=\frac{2}{\sqrt{3}} \operatorname{Ln}\left(1+z_{\mathrm{i} 0}^{\mathrm{H}} / R\right) \\
\varepsilon_{\mathrm{eq} i}^{\mathrm{B}}=\frac{2}{\sqrt{3}} \operatorname{Ln}\left(1+z_{\mathrm{i} 0}^{\mathrm{B}} / R\right)
\end{array} .\right.
$$

\subsection{Algorithm for the FLD prediction}

The general algorithm used to predict the FLD is based on the following three nested loops:

- For each strain path $\rho$ ranging from $-1 / 2$ to 1 (with increments $\Delta \rho$ of 0.1 ).

- For each initial band orientation $\theta_{0}$, spanning the admissible range of inclination angles (i.e., between $0^{\circ}$ and $90^{\circ}$ ), at user-defined intervals (here, we take intervals of $1^{\circ}$ ).

- For each time interval $\left[t_{0}, t_{0}+\Delta t\right]$, apply the implicit incremental algorithm described in Section 3.3 to integrate the governing equations detailed in Section 2. The application of this incremental integration scheme is stopped when the following criterion is satisfied:

$$
\Delta \varepsilon_{\mathrm{ST} Z \mathrm{Z}}^{\mathrm{B}} / \Delta \varepsilon_{\mathrm{ST} Z z}^{\mathrm{H}} \geq 10 .
$$

The strain component $\varepsilon_{\mathrm{STxx}}^{\mathrm{H}}$, thus obtained once the criterion (19) is satisfied, is considered as being the critical strain $\varepsilon_{x x}^{*}$ corresponding to the initial band inclination $\theta_{0}$ and strain path $\rho$. The factor 10 in Eq. (19) is rather arbitrary and any other relatively large positive value can be used without loss of accuracy. Indeed, the effect of this value on the predicted critical strain is minimal, since the absolute value of the band thickness strain rate $\dot{\varepsilon}_{\mathrm{ST} z z}^{\mathrm{B}}$ increases very rapidly towards infinity at incipient necking.

The lowest critical strain $\varepsilon_{\mathrm{xx}}^{*}$, over all initial angles $\theta_{0}$, and the corresponding current angle, define the necking limit strain $\varepsilon_{x x}^{\mathrm{L}}$ and the necking band orientation, respectively, for the current strain path $\rho$.

\subsection{Incremental integration of the governing equations}

The main purpose of this incremental algorithm is to integrate the equations governing the modified $\mathrm{M}-\mathrm{K}$ approach over a typical time increment $\left[t_{0}, t_{0}+\Delta t\right]$. In this aim, we assume that, at each integration point located in both zones, outside and inside the band, the following quantities are known at time $t_{0}$ :

- The coordinate through the thickness direction $z$.

- The equivalent strain $\varepsilon_{\text {eq }}$.

In order to simplify notations, the argument $t_{0}+\Delta t$ will be omitted hereinafter, with the implied understanding that the corresponding quantity is evaluated at $t_{0}+\Delta t$, unless otherwise indicated.

The increment $\Delta \varepsilon_{\mathrm{STzz}}^{\mathrm{B}}$ is chosen as loading parameter over $\left[t_{0}, t_{0}+\Delta t\right]$. It is denoted $\alpha$ (and is typically fixed to $0.001)$.

The increments $\Delta \varepsilon_{\mathrm{ST}}^{\mathrm{H}}$ and $\Delta \boldsymbol{\varepsilon}_{\mathrm{ST}}^{\mathrm{B}}$ are derived from the integration of Eqs. (11) and (12), respectively

$$
\begin{gathered}
\Delta \varepsilon_{\mathrm{ST}}^{\mathrm{H}}=\left(\begin{array}{cc}
\Delta \varepsilon_{\mathrm{ST} x \mathrm{x}}^{\mathrm{H}} & 0 \\
0 & \rho \Delta \varepsilon_{\mathrm{STxx}}^{\mathrm{H}}
\end{array}\right) . \\
\Delta \varepsilon_{\mathrm{ST}}^{\mathrm{B}}=\left(\begin{array}{cc}
\Delta \varepsilon_{\mathrm{ST} x \mathrm{x}}^{\mathrm{H}}+\Delta c_{1} n_{1} & (1 / 2)\left(\Delta c_{1} n_{2}+\Delta c_{2} n_{1}\right) \\
(1 / 2)\left(\Delta c_{1} n_{2}+\Delta c_{2} n_{1}\right) & \rho \Delta \varepsilon_{\mathrm{ST} x \mathrm{H}}^{\mathrm{H}}+\Delta c_{2} n_{2}
\end{array}\right)
\end{gathered}
$$

It is noteworthy that Eq. (21) has been derived from Eq. (12) by implicitly assuming that, all along the time increment, the components of vector $\vec{n}$ keep their initial values at $t_{0}$. 
Furthermore, Eq. (21) in conjunction with the incompressibility condition leads to the following relation:

$$
(1+\rho) \Delta \varepsilon_{\mathrm{STxx}}^{\mathrm{H}}+\Delta c_{1} n_{1}+\Delta c_{2} n_{2}=-\Delta \varepsilon_{\mathrm{STzz}}^{\mathrm{B}} .
$$

In the band, the position of the different integration points at $t_{0}+\Delta t$ can be obviously expressed in terms of $\Delta \varepsilon_{\mathrm{STzZ}}^{\mathrm{B}}$

$$
\forall i=1, \ldots, N_{\mathrm{p}}: z_{i}^{\mathrm{B}}=z_{i}^{\mathrm{B}}\left(t_{0}\right) \mathrm{e}^{\Delta \varepsilon_{\mathrm{STzZ}}^{\mathrm{B}}} .
$$

By using Eqs. (10) and (23), the increment of $\boldsymbol{\varepsilon}_{\mathrm{BE}}^{\mathrm{B}}$ over $\left[t_{0}, t_{0}+\Delta t\right]$ for the different integration points within the band is derived as

$$
\forall i=1, \ldots, N_{\mathrm{p}}: \Delta \boldsymbol{\varepsilon}_{\mathrm{BEi}}^{\mathrm{B}}=\left(\begin{array}{cc}
\operatorname{Ln}\left(\frac{R+z_{i}^{\mathrm{B}}}{R+z_{i}^{\mathrm{B}}\left(t_{0}\right)}\right) & 0 \\
0 & 0
\end{array}\right) .
$$

The position at $t_{0}+\Delta t$ of the integration points located outside the band can be determined by the following equation:

$$
\forall i=1, \ldots, N_{\mathrm{p}}: z_{i}^{\mathrm{H}}=z_{i}^{\mathrm{H}}\left(t_{0}\right) \mathrm{e}^{\Delta \varepsilon_{\mathrm{ST} Z Z}^{\mathrm{H}}},
$$

where $\Delta \varepsilon_{\mathrm{STzz}}^{\mathrm{H}}$ is determined by applying the incompressibility condition

$$
\Delta \varepsilon_{\mathrm{STzz}}^{\mathrm{H}}=-(1+\rho) \Delta \varepsilon_{\mathrm{ST} \mathrm{xx}}^{\mathrm{H}} .
$$

The increment of $\boldsymbol{\varepsilon}_{\mathrm{BE}}^{\mathrm{H}}$ over $\left[t_{0}, t_{0}+\Delta t\right]$ for the different integration points located outside the band is determined by a relation very similar to Eq. (24)

$$
\forall i=1, \ldots, N_{\mathrm{p}}: \Delta \boldsymbol{\varepsilon}_{\mathrm{BEi}}^{\mathrm{H}}=\left(\begin{array}{cc}
\operatorname{Ln}\left(\frac{R+z_{i}^{\mathrm{H}}}{R+z_{i}^{\mathrm{H}}\left(t_{0}\right)}\right) & 0 \\
0 & 0
\end{array}\right) .
$$

The increment over $\left[t_{0}, t_{0}+\Delta t\right]$ of the strain tensors $\boldsymbol{\varepsilon}^{\mathrm{H}}$ and $\boldsymbol{\varepsilon}^{\mathrm{B}}$ at the different integration points located outside and in the band zone, respectively, is finally determined as follows:

$$
\forall i=1, \ldots, N_{\mathrm{p}}:\left\{\begin{array}{l}
\Delta \boldsymbol{\varepsilon}_{\mathrm{i}}^{\mathrm{H}}=\Delta \boldsymbol{\varepsilon}_{\mathrm{ST}}^{\mathrm{H}}+\Delta \boldsymbol{\varepsilon}_{\mathrm{BEi}}^{\mathrm{H}} \\
\Delta \boldsymbol{\varepsilon}_{\mathrm{i}}^{\mathrm{B}}=\Delta \boldsymbol{\varepsilon}_{\mathrm{ST}}^{\mathrm{B}}+\Delta \boldsymbol{\varepsilon}_{\mathrm{BEi}}^{\mathrm{B}}
\end{array},\right.
$$

where the expressions of $\Delta \boldsymbol{\varepsilon}_{\mathrm{ST}}^{\mathrm{H}}, \Delta \boldsymbol{\varepsilon}_{\mathrm{BEi}}^{\mathrm{H}}, \Delta \boldsymbol{\varepsilon}_{\mathrm{ST}}^{\mathrm{B}}$, and $\Delta \boldsymbol{\varepsilon}_{\mathrm{BE} \mathrm{i}}^{\mathrm{B}}$ are given in Eqs. (20), (27), (21), and (24), respectively. Accordingly, for a given strain path $\rho$, the strain increments $\Delta \varepsilon_{\mathrm{i}}^{\mathrm{H}}$ and $\Delta \boldsymbol{\varepsilon}_{\mathrm{i}}^{\mathrm{B}}$ are functions of three scalar unknowns: $\Delta \varepsilon_{\mathrm{ST} \mathrm{Xx}}^{\mathrm{H}}, \Delta c_{1}$ and $\Delta c_{2}$.
The expression of the deviatoric stress $\mathbf{S}$ at the different integration points inside and outside the band is derived from the incremental form of Eq. (5)

$$
\mathbf{S}=\frac{2 K \varepsilon_{\mathrm{eq}}^{n}}{3 \Delta \varepsilon_{\mathrm{eq}}} \Delta \boldsymbol{\varepsilon}
$$

Combining Eqs. (3) and (7), one can easily derive the expression of the Cauchy stress tensor $\boldsymbol{\sigma}$ at the different integration points

$$
\boldsymbol{\sigma}=\mathbf{S}+\left(S_{\mathrm{xx}}+S_{\mathrm{yy}}\right) \mathbf{I}_{2}
$$

The above expression for $\boldsymbol{\sigma}$ is then inserted in the approximation (16), which in turn is inserted in the equilibrium equation (14). Analyzing the previous developments, Eq. (14) may be regarded as a system of two equations with three scalar unknowns: $\Delta \varepsilon_{\mathrm{STx}}^{\mathrm{H}}, \Delta c_{1}$ and $\Delta c_{2}$. By considering Eq. (14) along with Eq. (22), a system of three scalar equations with the three above unknowns is obtained. This system should be solved iteratively by using the Newton-Raphson method in order to determine the above-mentioned scalar unknowns. Ultimately, the determination of these unknowns allows computing the different quantities at $t_{0}+\Delta t$ : the distance of the different integration points to the neutral axis of the sheet, the distribution of the equivalent strain through the thickness in both zones, and the band orientation. In particular, the values of the strain components $\Delta \varepsilon_{\mathrm{STzz}}^{\mathrm{H}}$ and $\varepsilon_{\mathrm{STxx}}^{\mathrm{H}}$ (the latter being equal to $\left.\varepsilon_{\mathrm{ST} \mathrm{xx}}^{\mathrm{H}}\left(t_{0}\right)+\Delta \varepsilon_{\mathrm{STXx}}^{\mathrm{H}}\right)$ are of special interest; the former being required for the application of the necking criterion (19), while the latter is needed in the algorithm of Section 3.2 .

\section{Prediction results}

A DP600 sheet steel with the hardening parameters $K=945 \mathrm{MPa}$ and $n=0.16$ is used in the following simulations. The initial thicknesses $h_{0}^{\mathrm{H}}$ and $h_{0}^{\mathrm{B}}$ are fixed to $1 \mathrm{~mm}$ and $0.99 \mathrm{~mm}$, respectively. The number of integration points $N_{\mathrm{p}}$ is set to 11 . To simplify the notations used in the following results, the stretching strains $\varepsilon_{\mathrm{ST} \mathrm{xx}}^{\mathrm{H}}, \varepsilon_{\mathrm{ST} \mathrm{yy}}^{\mathrm{H}}, \varepsilon_{\mathrm{ST} \mathrm{zz}}^{\mathrm{H}}$, and $\varepsilon_{\mathrm{ST} \mathrm{zz}}^{\mathrm{B}}$ are simply denoted $\varepsilon_{11}^{\mathrm{H}}, \varepsilon_{22}^{\mathrm{H}}, \varepsilon_{33}^{\mathrm{H}}$, and $\varepsilon_{33}^{\mathrm{B}}$, respectively. In fact, the latter simplified notations are more common for the presentation of results corresponding to FLD predictions.

Figure 2 shows the onset of localized necking in the case of plane-strain state $(\rho=0)$. In this figure, three bending radii are considered: $R=3 \mathrm{~mm} ; R=10 \mathrm{~mm} ; R$ $=100 \mathrm{~mm}$. The results corresponding to a classical flat sheet are also included in this figure. The occurrence of strain localization is predicted when the ratio $\Delta \varepsilon_{33}^{\mathrm{B}} / \Delta \varepsilon_{33}^{\mathrm{H}}$ exceeds 10 , as stated by criterion (19). It is clear from this figure that the limit strain increases with the bending 
radius $R$. This means that bending has as effect to accelerate the occurrence of localized necking, especially when the bending radius is small. For large values of the bending radius $R$ (e.g., more than $100 \mathrm{~mm}$ ), the bending effect is very limited. In such conditions, the prediction with high bending radius is almost the same as that corresponding to a flat sheet, as observed in Figure 2.

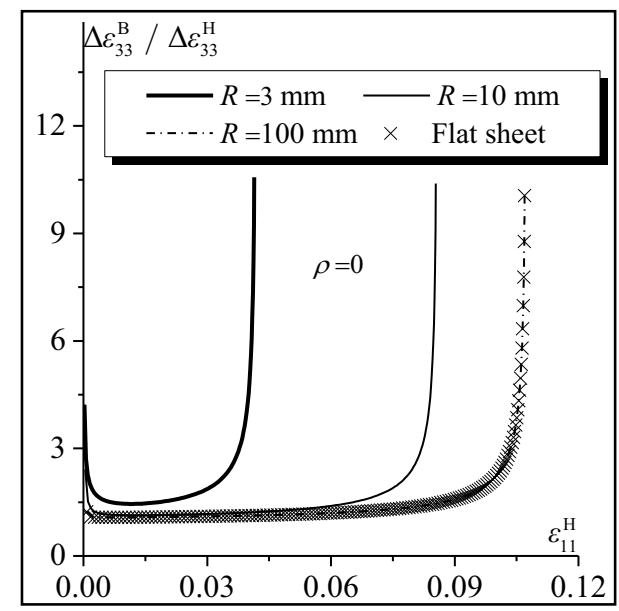

Figure 2. Illustration of the onset of localized necking in the case of plane-strain state $(\rho=0)$

The evolution of the critical strain $\varepsilon_{11}^{*}$ as a function of the curvature $1 / R$ is plotted in Figure 3 for four representative strain paths $(\rho=-0.5 ; \rho=0 ; \rho=0.5$; and $\rho$ $=1)$. The initial band orientation is taken equal to $30^{\circ}$ for the first strain path (which is different from the necking band orientation), and $0^{\circ}$ for the other strain paths. For the different strain paths, the effect of curvature on necking acceleration is obvious, especially for the uniaxial tensile state $(\rho=-0.5)$. The same trend was observed in [8]. Small vertical lines indicate the results for the bending radii of $100 \mathrm{~mm}, 10 \mathrm{~mm}$, and $3 \mathrm{~mm}$.

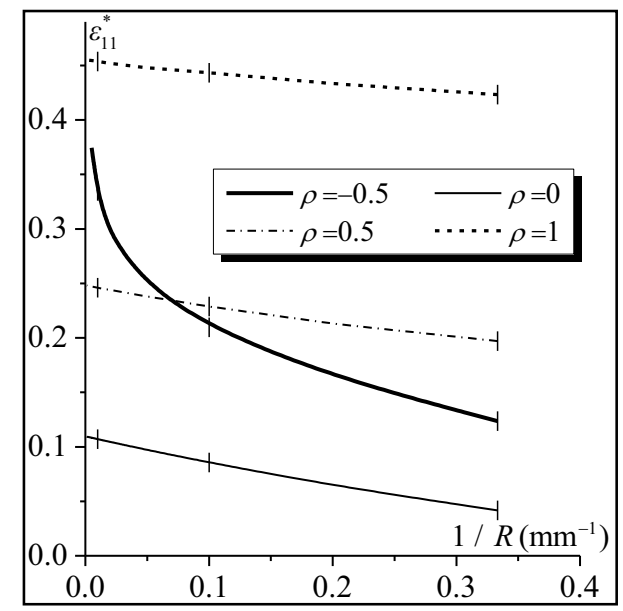

Figure 3. Evolution of the critical strain $\varepsilon_{11}^{*}$ as a function of the curvature $1 / R$

The effect of bending radius on the evolution of the critical strain $\varepsilon_{11}^{*}$, as a function of the initial band orientation $\theta_{0}$, is investigated in Figure 4 for the case of uniaxial tensile strain path. Note that the necking band orientation is not perpendicular to the major strain direction, whatever the value of the bending radius $R$. Indeed, the critical strain $\varepsilon_{11}^{*}$ decreases with the initial band orientation $\theta_{0}$ until reaching its lowest value, which corresponds to the limit strain $\varepsilon_{11}^{\mathrm{L}}$, and increases afterwards. This result is common to all negative strain paths, as demonstrated in Figure 5. However, for positive strain paths, the necking band is perpendicular to the major strain direction. These observations are classical for flat sheets when bending effects are not considered. The effect of bending radius on the band orientation $\theta$ is not very significant, as shown in Figure 5.

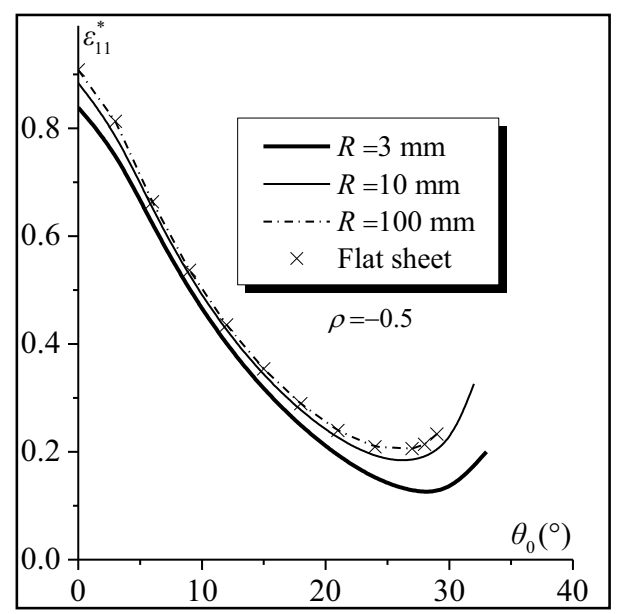

Figure 4. Effect of the bending radius on the evolution of the critical strain $\varepsilon_{11}^{*}$ as a function of the initial band orientation $\theta_{0}$ for uniaxial tensile strain path

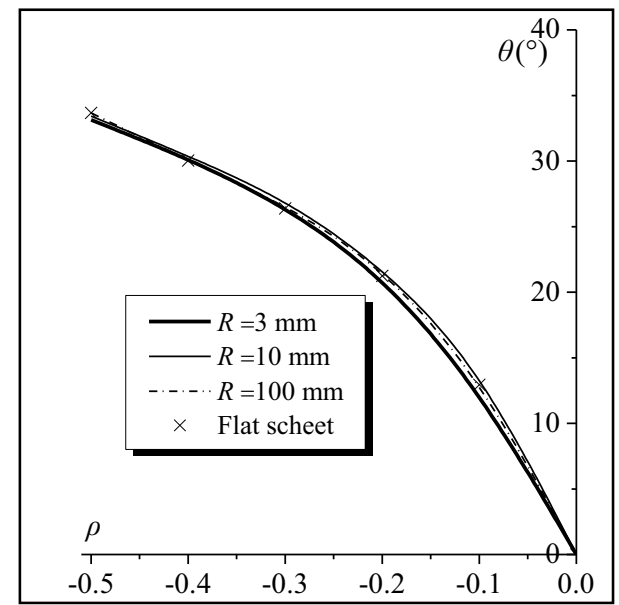

Figure 5. Effect of the bending radius on the evolution of the necking band orientation $\theta$ as a function of the strain path $\rho$

The results presented in Figure 4 are extended in Figure 6 to the whole range of strain paths, where the effect of bending radius on the location and shape of FLDs is analyzed. It appears from this figure that the bending effect is more important in the left-hand side of the FLD than in its right-hand side. The predictions reported in this figure confirm once again that the 
classical FLD results, corresponding to a flat sheet, are naturally recovered in the limit of very large curvature radius.

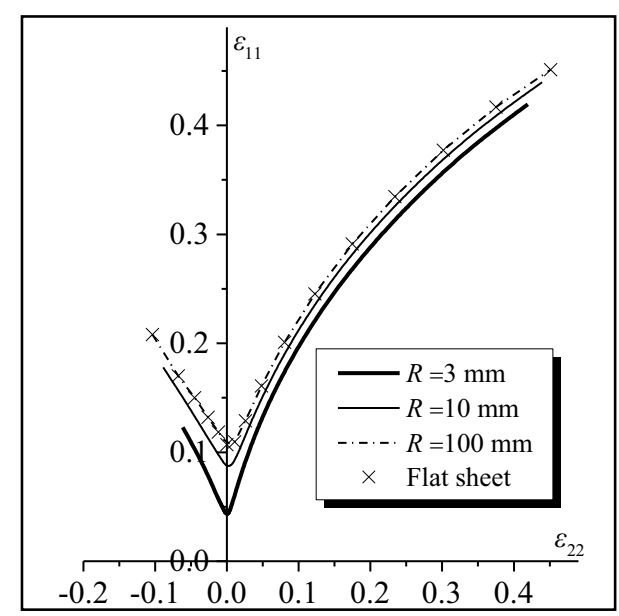

Figure 6. Effect of the bending radius on the location and the shape of FLDs

\section{Concluding remarks}

A numerical tool has been developed in this paper for the prediction of localized necking in sheet metals undergoing combined bending-stretching loadings. This model may be useful for understanding how the bending effects involved in forming processes would affect sheet metal formability. Contrary to previous models, specifically devoted to this type of combined loadings, the current model is able to predict the occurrence of localized necking for the whole range of strain paths. In the current approach, the mechanical behavior of the sheet metal is described by the rigid-plastic flow theory, while localized necking prediction is based on the imperfection approach. From the numerical predictions obtained by applying this tool, it is concluded that the addition of bending loading to traditional stretching tends to accelerate the occurrence of localized necking.

\section{References}

1. S.P. Keeler, W.A. Backofen, Trans. ASM 56, 25 (1963)

2. G.M. Goodwin, Metallurgia Italiana 60, 767 (1968)

3. S.L. Chiu, J. Leu, P.S. Ho, J. Appl. Phys. 76, 5136 (1994)

4. Z. Marciniak, K. Kuczynski, Int. J. Mech. Sci. 9, 609 (1967)

5. J.W. Hutchinson, K.W. Neale, Koistinen, D.P., Wang, N.M. (Eds.), Mechanics of Sheet Metal Forming. Plenum, 127 (1978)

6. M. Shi, J. Gerdeen, J. Mater. Shaping Technol. 9, 253 (1991)

7. S. Sriram, H. Yao, N. Ramisetti, J. Manuf. Sci. Eng. 134, 031003 (2012)
8. J. He, Z.C. Xia, S. Li, D. Zeng, J. Manuf. Sci. Eng. 135, 227 (2013)

9. J. He, Z.C. Xia, D. Zeng, S. Li, J. Eng. Mat. Tech. 135, 031009 (2013)

10. R. Safdarian, Mech. Res. Commun. 67, 47 (2015) 